\title{
1 Genome-wide association uncovers the genetic architecture of tradeoff between flowering date and yield components in sesame
}

$4 \quad$ Idan Sabag ${ }^{1,2}$, Gota Morota ${ }^{2,3}$, and Zvi Peleg, ${ }^{1 *}$

5

$6{ }^{1}$ The Robert H. Smith Institute of Plant Sciences and Genetics in Agriculture, The Hebrew

7 University of Jerusalem, Rehovot 7610001, Israel.

$8 \quad{ }^{2}$ Department of Animal and Poultry Sciences, Virginia Polytechnic Institute and State University,

9 Blacksburg, Virginia 24061 USA.

$10{ }^{3}$ Center for Advanced Innovation in Agriculture, Virginia Polytechnic Institute and State 11 University, Blacksburg, Virginia 24061 USA.

12

13

$14 *$ Corresponding author (zvi.peleg@ mail.huji.ac.il) 


\section{Abstract}

17 Unrevealing the genetic makeup of crop morpho-agronomic traits is essential for improving yield 18 quality and sustainability. Sesame (Sesamum indicum L.), one of the oldest oil-crops in the world,

19 which despite its economical and agricultural importance, is an 'orphan crop-plant' that undergone

20 limited modern selection, thus, preserving wide genetic diversity. Here we harnessed this natural

21 variation in a newly developed sesame panel (SCHUJI) to perform genome-wide association

22 studies for morpho-agronomic traits under the Mediterranean climate conditions. Field-based

23 phenotyping of the SCHUJI panel across two seasons exposed wide phenotypic variation for all

24 traits. Using 20,294 single-nucleotide polymorphism markers, we detected 50 genomic signals

25 associated with these traits. Major genomic region on LG2 was associated with flowering date and

26 yield-related traits, exemplified the key role of the flowering date on productivity. Our results shed

27 light on the genetic architecture of flowering date and its interaction with yield components in sesame and may serve as a basis for future sesame breeding programs in the Mediterranean basin.

Abbreviations: Best linear unbiased estimate (BLUE), candidate genes (CGs), flowering date (FD), genome-wide association studies (GWAS), height to the first capsule (HTFC), number of

37 branches per plants (NBPP), plant height $(\mathrm{PH})$, reproductive index (RI), reproductive zone (RZ),

38 single nucleotide polymorphism (SNP), seed number per plant (SNPP), seed yield per plant 39 (SYPP), thousand-seed weight (TSW). 


\section{INTRODUCTION}

42 Sesame (Sesamum indicum L.), one of the oldest oil-crops in the world, was domesticated about

43 5,500 years ago from Sesamum indicum subsp. malabaricum (Bedigian, 2015) in the Indian sub-

44 continent. Sesame is an annual diploid $(2 n=2 x=26)$ species, which belongs to the Sesamum genus

45 from the Pedaliaceae family. Its seeds are comprised of oil (45-60\%), proteins (18-25\%),

46 carbohydrate (3-25\%), and rich with essential vitamins and mineral-nutrients (Anilakumar et al.,

47 2010; Teboul et al., 2020). The seeds are being used for an array of products in the food (e.g.,

48 high-quality oil, tahini paste, and cooking and backing) and pharmaceutical industries (Mushtaq,

49 2020). At present, sesame is cultivated mainly in developing countries, with annual seed

50 production of 6.7 million tons (http://www.fao.org/faostat/en/\#data/QC). The global shift toward

51 healthier and more nutritional plant-based food products lead to significantly increased demand for

52 sesame seeds and derivative products. However, despite its economical and agricultural

53 importance, sesame is considered an 'orphan crop-plant' and has been subjected to limited

54 agronomical and scientific research.

55 Sesame is a short-day erect plant with an indeterminate florescence and simple or branching 56 rigid stem. Its growth period ranges usually from 12-18 weeks, with flowering (i.e., the transition

57 from vegetative to reproductive phase) begins about 30-40 (early) to 70-80 (late) days after sowing

58 (Langham, 2007). This variation in flowering time could affect the crop adaption to specific agro-

59 system conditions. As the blooming period continues until plant maturation, the flowering date

60 plays a crucial role in both plant architecture and yield components. Sesame yield components

61 include the number of plants per unit area, number of branches per plant, number of capsules per

62 leaf axil, seeds per capsule, and seed weight (York \& Garden, 2017). Complex tradeoffs between

63 yield component traits have been shown to significantly affect the final seed yield (Gadri et al.,

64 2020). Branching habit and number of capsules per leaf axil were shown to support higher seed

65 yield (Mei et al., 2017), whereas dwarf mutants (i.e., small plant height) negatively affect seed 66 weight (Miao et al., 2020).

67 The advent of next-generation sequencing and genotyping by sequencing (GBS) technologies has provided a means for examining genetic diversity and population structure of crop-plants, which can facilitate the genetic dissection of agronomic traits and integrate them in breeding

70 programs. Genome-wide association studies (GWAS) are a promising approach that connects

71 phenotypic variation and genomic data (i.e., genetic markers) to detect genomic regions underlying 
72 complex traits (Tibbs Cortes et al., 2021). GWAS were applied successfully for various crop73 plants, such as bread wheat (Triticum aestivum L.; Guo et al., 2017), maize (Zea mays L.; Li et al., 74 2012), rice (Oryza sativa L.; Zhao et al., 2011), and soybean (Glycine max L.; Sonah, 2015). In 75 sesame, GWAS were used for the identification of genomic regions associated with response to 76 biotic (Asekova et al., 2021) and abiotic (Li et al., 2018; Dossa et al., 2019) stresses, as well as 77 yield-related traits (Zhou et al., 2018). The relatively small genome size ( $375 \mathrm{Mbps})$, the recent 78 development of genomic resources and rich genetic diversity make sesame an ideal model crop for 79 genetic investigation (Dossa et al., 2017).

Here we harness the natural variation among geographically distributed sesame germplasm to underpin the genetic architecture of morpho-agronomic and yield-related traits. Our working hypothesis was that as a consequence of minimal artificial selection processes (associated with modern breeding), sesame preserved rich genetic and phenotypic diversity that will enable detection of novel genomic regions conferring agronomical important traits. The aims of the current study were to $(\boldsymbol{i})$ characterize the genetic diversity in the newly established sesame collection, (ii) detect genomic regions contributing to the phenotypic performance, and (iii) infer the genetic associations between traits. Our findings shed new light on the interaction between flowering date, morpho-physiological traits, and yield components in sesame.

\section{MATERIALS AND METHODS}

\subsection{Plant material and experimental design}

A panel of 184 sesame genotypes from the Hebrew University of Jerusalem sesame collection was assembled (SCHUJI panel, hereafter) according to their geographical origins to capture the whole sesame genepool genetic diversity (Supplemental Table S1). The plants were grown over two growing seasons (2018 and 2020) at the experimental farm of Hebrew University of Jerusalem in Rehovot, Israel $\left(34^{\circ} 47^{\prime} \mathrm{N}, 31^{\circ} 54^{\prime} \mathrm{E} ; 54 \mathrm{~m}\right.$ above sea level). The soil at this location is brown-red degrading sandy loam (Rhodoxeralf) composed of $76 \%$ sand, $8 \%$ silt, and $16 \%$ clay. In the 2018 growing season, a complete random factorial (genotypes) block design with seven replicates was employed. Each block consisted of 184 plots sown as single row, with six plants, $15 \mathrm{~cm}$ apart (1- 
102

103

104

105

106

107

108

109

110

111

112

113

114

115

116

117

118

119

120

121

122

123

124

125

126

plants were used for phenotypic characterization and at the end of the experiment, they were harvested to estimate yield components. In the 2020 growing season, the same experimental design was employed with five replicates per genotype. The plot size was $2.6 \mathrm{~m} \times 0.8 \mathrm{~m}(15 \mathrm{~cm}$ between plants) with three rows per plot. Five representing plants from the middle row were used for phenotypic evaluation at maturity and harvested to obtain yield components. In both seasons, the field was treated with fungicides and pesticides to avoid the development of fungal pathogens or insect pests and was weeded manually once a week.

\subsection{Phenotypic measurements}

Phenotypes were recorded during the whole sesame growing season for each plot. Flowering date (FD) was evaluated visually when $50 \%$ of the plants in each plot had at least one open flower. Height to the first capsule (HTFC) and plant height $(\mathrm{PH})$ were measured at maturity from the soil surface to the first capsule and the plant tip, respectively. The reproductive zone of the main stem (RZ) was calculated as the delta between PH and HTFC, and the reproductive index (RI) was calculated as the ratio between RZ and $\mathrm{PH}(\mathrm{RZ} / \mathrm{PH})$. Before harvest, the number of branches per plant (NBPP) were counted as an average of all individual plant in a plot. At physiological maturity, three plants (2018) and five plants (2020) from each plot were harvested, and sun-dried. The samples were threshed using the laboratory threshing machine (LD 350, WinterSteiger, Reid, Austria). Seeds were counted using the seeds counting machine (Data Count S25, Data Technologies) and weighted in analytical lab weight to obtain seed number per plant (SNPP), seed yield per plant (SYPP), and thousand-seed weight (TSW) for each plot.

\subsection{Statistical analysis of phenotypic data}

The JMP ver. 15 pro statistical package (SAS Institute, Cary, NC, USA) and R (R Core Team, 2020) was used for all statistical analyses with a significant threshold of 5\%. First, we calculated the best linear unbiased estimate (BLUE) for every trait for each genotype per year and for both years using the lme4 R package (Bates et al., 2015). The mixed linear model for BLUE per year was fitted according to the formula:

$$
y_{i o}=\mu+g_{i}+b_{o}+\epsilon,
$$


132

133

where $y_{i o}$ is the phenotypic observation for the $i$ th genotype in the oth block, $\mu$ is the intercept, $g_{i}$ is the genotype fixed effect, $b_{o}$ is the block random effect, and $\epsilon$ is the model residuals.

The BLUE for the combined data from the two years was calculated according to the formula:

where $y_{i k o}$ is the phenotypic observation for the $i$ th genotype in the $k$ th year and the $o$ th block, $\mu$ is the intercept, $g_{i}$ is the genotype fixed effect, $e_{k}$ is the random effect of year, $(g e)_{i k}$ is the random effect of genotype-by-year interaction, $b_{k o}$ is the random effect of block nested within year, and $\epsilon$ is the model residual. For the calculation of heritability, we fitted the same mixed model as above, with the expectation of genotype, that considered as random effect. A broad-sense heritability was calculated on the entry-mean basis according to Schmidt et al. (2019) using the estimated variance components:

$$
y_{i k o}=\mu+g_{i}+e_{k}+(g e)_{i k}+b_{k o}+\epsilon,
$$

$$
\text { estimated variance components: }
$$$$
H^{2}=\frac{\sigma_{G}^{2}}{\sigma_{G}^{2}+\frac{\sigma_{G \times Y}^{2}}{n_{Y}}+\frac{\sigma_{\epsilon}^{2}}{n_{Y} n_{r}}},
$$

where $\sigma_{G}^{2}, \sigma_{G \times Y}^{2}$ and $\sigma_{\epsilon}^{2}$ are the genetic, genotype-by-year interaction, and residual variances, respectively, and $n_{Y}$ is the number of years (2) and $n_{r}$ is the average number of replicates across years (6). The significance of variance components was evaluated by the likelihood ratio test using the lmerTest R package (Kuznetsova et al., 2017).

We performed GWAS for each data set (referred to as 2018 data, 2020 data, and combined data, hereafter). The combined data set was used to infer phenotypic and genomic correlations and to conduct principal components analysis (PCA), k-means clustering, and genomic heritability estimation. K-means clustering analysis was applied using the factoextra R package (Kassambara and Mundt, 2020) on the centered and scaled values of each trait. Both PCA and clusters plot was drawn in the JMP (ver.15pro) statistical package. Density plots and a correlation matrix were constructed using the ggplot2 (Wickham, 2016) and the corrplot (Wei and Simko, 2017) R packages. 


\subsection{Genotyping and preparation of marker data set}

162 Genomic DNA was extracted from young leaf tissues with a modified CTAB method (Doyle, 1987). We generated GBS data using the procedure described in Elshire et al. (2011), with minor changes: $100 \mathrm{ng}$ of genomic DNA and $3.6 \mathrm{ng}$ of total adapters were used. Genomic DNA was restricted with ApeKI enzyme and the library was amplified with 18 PCR cycles. We used the Zhongzhi No. 13 (https://www.ncbi.nlm.nih.gov/assembly/GCF_000512975.1) reference genome to perform reference-based SNP calls based on the STACKS 2.3 pipeline (http://catchenlab.life.illinois.edu/stacks). These analyses yielded 90,542 single nucleotide polymorphisms markers (SNPs) in total including unknown scaffolds and chloroplast genome. Markers on unknown scaffolds and chloroplast genome were removed and all the markers were

171 filtered to depth quality of 3 using TASSEL ver. 5.0 (Bradbury et al., 2007). Imputation of missing genotypes was performed by BEAGLE ver. 5 (Browning \& Browning, 2007). We also removed markers that were tightly linked $\left(r^{2}=0.99\right)$ using PLINK (Purcell et al., 2007). Polymorphic sites

174 with $<5 \%$ minor allele frequency and $>20 \%$ heterozygosity were filtered out by PLINK and TASSEL, respectively. The remaining 20,294 SNPs were used for further analysis.

\subsection{Population structure, kinship, and linkage disequilibrium}

178 We used PCA and a centered identity-by-state matrix $(\mathrm{G})$ constructed from TASSEL (Bradbury et 179 al., 2007) to infer population structure in the sesame panel. The ADMIXTURE software (Alexander et al., 2009) was used to estimate a Q-matrix, which is the ancestry among the accessions. To select the number of subpopulations (K), we ran the software from $\mathrm{K}=1$ to 10 with

182 five-fold cross-validation. This analysis outputs the cross-validation error (\%) for a given K. The number of subpopulations was determined as $\mathrm{K}$ that produced the lowest cross-validation error. The lowest value of cross-validation error was achieved with $\mathrm{K}=7$ (Supplemental Table S2), and the Q-matrix was constructed with 1,000 bootstrapping. The results from the PCA analysis and ADMIXTURE software outputs were plotted using the ggplot $2 \mathrm{R}$ package. The fixation index ( $\left.\mathrm{F}_{\mathrm{st}}\right)$ among the seven subpopulations was calculated with VCFtools (Danecek et al., 2011) according to Weir \& Cockerham (1984). Genome-wide linkage disequilibrium (LD) was obtained through pairwise correlations between markers with a sliding window of 10 markers using PLINK (Purcell et al., 2007). The correlation between markers $\left(r^{2}\right)$ was plotted against their physical positions (i.e., 
191

192

193

194

195

196

197

198

199

200

201

202

203

204

205

206

207

208

209

210

211

212

213

214

215

216

217

218

base pairs) and the extent of LD pattern and decay was obtained by fitting a non-linear model according to Hill \& Weir (1988) as described in Marroni et al. (2011). We used a value (in base pairs) in which LD halves from its initial value for defining haplotypes and mining for candidate genes (CG) around significant markers.

\subsection{Genomic heritability and genomic correlations}

Genomic heritability estimates and genomic correlations were inferred using the BGLR R package (Pérez \& De Los Campos, 2014). To obtain genomic heritability for each trait, we fitted a Bayesian univariate genomic best linear unbiased prediction using the equation:

$$
\mathbf{Y}=\mu+\mathbf{X b}+\mathbf{u}+\boldsymbol{\epsilon}
$$

where $\mathbf{Y}$ is the vector of single-trait BLUE phenotypes, $\mu$ is the intercept, $\mathbf{X}$ is a design matrix for fixed effects, $\mathbf{b}$ is the vector of fixed effects containing three PCs to account for population structure, $\mathbf{u}$ is the vector of random effects, and $\boldsymbol{\epsilon}$ is a vector of the model residuals. The following distributions were assumed for random effects:

$$
\left(\begin{array}{l}
\boldsymbol{\mu} \\
\boldsymbol{\varepsilon}
\end{array}\right) \sim \mathrm{N}\left[\left(\begin{array}{l}
0 \\
0
\end{array}\right),\left(\begin{array}{cc}
\mathbf{G} \sigma_{\mathrm{u}}^{2} & 0 \\
0 & \mathbf{I} \sigma_{\epsilon}^{2}
\end{array}\right)\right]
$$

where $\mathbf{G}$ represents the first genomic relationship matrix of VanRaden (2008), I is the identity matrix, and $\sigma_{\mathrm{u}}^{2}$ is the additive genomic variance explained by genetic markers, and $\sigma_{\epsilon}^{2}$ is the model residuals. Genomic heritability was calculated as: $h^{2}=\frac{\sigma_{\mathrm{u}}^{2}}{\sigma_{\mathrm{u}}^{2}+\sigma_{\epsilon}^{2}}$.

Genomic correlations were estimated using the multivariate version of the model described above, where $\mathbf{Y}$ is the vector of multi-trait phenotypes. The following distributions were assumed for random effects:

$$
\left(\begin{array}{l}
\boldsymbol{\mu} \\
\boldsymbol{\varepsilon}
\end{array}\right) \sim \mathrm{N}\left[\left(\begin{array}{l}
0 \\
0
\end{array}\right),\left(\begin{array}{cc}
\Sigma_{\mu} \otimes \mathbf{G} & 0 \\
0 & \Sigma_{\epsilon} \otimes \mathbf{I}
\end{array}\right)\right]
$$


where $\Sigma \mathrm{u}$ and $\Sigma$ e refer to the genetic and residual variance-covariance matrices, respectively, and

$221 \otimes$ is the Kronecker product.

Genomic correlations between traits were derived from the genomic variance-covariance matrix as:

$$
\operatorname{corr}\left(\mathbf{u}_{1}, \mathbf{u}_{2}\right)=\frac{\sigma_{\mathbf{u}_{1} \mathbf{u}_{2}}^{2}}{\sqrt{\sigma_{\mathbf{u}_{1}}^{2}} \sqrt{\sigma_{\mathbf{u}_{2}}^{2}}}
$$

where $\mathbf{u}_{1}$ and $\mathbf{u}_{2}$ are the breeding values of traits $\mathbf{Y}_{1}$ and $\mathbf{Y}_{2}, \sigma_{\mathbf{u}_{1} \mathbf{u}_{2}}^{2}$ is the additive genomic respectively.

\subsection{Association mapping}

232 To identify genomic regions associated with the traits of interest, we used a mixed linear model of 233 Henderson (1975) coupled with the first three PCs and G matrices to account for population 234 structure and relatedness among individuals, respectively, using the rrBLUP R package (Endelman, 2011). We fit an additive single-marker GWAS model as the following:

$$
\mathbf{Y}=\mathbf{X b}+\mathbf{Z u}+\boldsymbol{\epsilon}
$$

239 where $\mathbf{Y}$ is the vector of phenotypes, $\mathbf{b}$ is the vector of fixed effects including an SNP and 3 PCs, $240 \mathbf{u}$ is a vector of random additive genetic effects with mean zero and variance-covariance $\boldsymbol{G} \sigma_{u}^{2}, \mathbf{X}$ 241 and $\mathbf{Z}$ are the known incidence matrices, and $\mathbf{\epsilon}$ is the vector of residuals (Yu et al., 2006). The $P$ 242 value threshold $\left(1.551509 \times 10^{-5}\right)$ was determined by calculating the number of effective 243 independent tests (Meff) as described in Li \& Ji (2005) with the following formula: $P=1$ - (1$2440.05)^{\wedge}(1 / \mathrm{Meff})$, where $P$ is the genome-wide $P$-value threshold and 0.05 is the desired level of 245 significance, and Meff = 3,306. 


\subsection{Haplotypes estimation and candidate gene analysis}

248 Haplotypes encompassing associated markers that corresponded to the LD-decay pattern in the 249 SCHUJI panel was constructed in TASSEL version 5.0 (Bradbury et al., 2007). We included only 250 haplotypes with a frequency greater than $5 \%$ and only genotypes that were homozygous in all the 251 genetic markers within a haplotype. The effects of haplotypes on the phenotypic variation were 252 estimated using analysis of variance (JMP ver. 15) at a significant level of 5\%. Phenotypic response 253 was the BLUE of 2018 and 2020 growing seasons. A haplotype plot was produced using the 254 Rainclouds R package (Allen et al., 2021). We used LD-decay for mining CGs around significant 255 SNPs and analyzed them according to the Zhongzhi No. 13 reference genome 256 (https://www.ncbi.nlm.nih.gov/assembly/GCF_000512975.1).

\section{RESULTS}

\subsection{High phenotypic diversity among the sesame panel}

261 To test the level of phenotypic diversity among the newly established SCHUJI panel, we 262 characterized the sesame under Mediterranean basin conditions over two seasons. In general, the 263 SCHUJI panel exhibited rich variation for phenological, plant architecture, and yield components 264 (Fig. 1; Supplemental Table S3; Fig. S1). Flowering date (FD) showed a similar pattern across 265 years and spread along most of the growing season, ranging between 39.86 to 76 and 39.2 to 70 266 days after sowing (DAS) for 2018 and 2020, respectively. In both years (2018 and 2020), most 267 genotypes (135 and 146) flowered before 55 DAS (Fig. 1A). Plant architecture traits showed a 268 wider range in the 2020 than 2018 seasons for HTFC (29-140 vs. 36-198 cm; Fig. 1B), PH (80-177 269 vs. 103-251 cm; Fig. 1C), and RZ (20 to 104 vs. 28 to 141cm; Fig. S1). In contrast, RI exhibited a 270 similar range in both seasons (0.13-0.73 vs. 0.12-0.76; Fig. 1D). Most yield components showed 271 a similar pattern across years (SYPP and TSW) except SNPP which exhibited a much wider variation in 2020 (21-7719 vs. 508-13591) (Fig. 1F-H). 

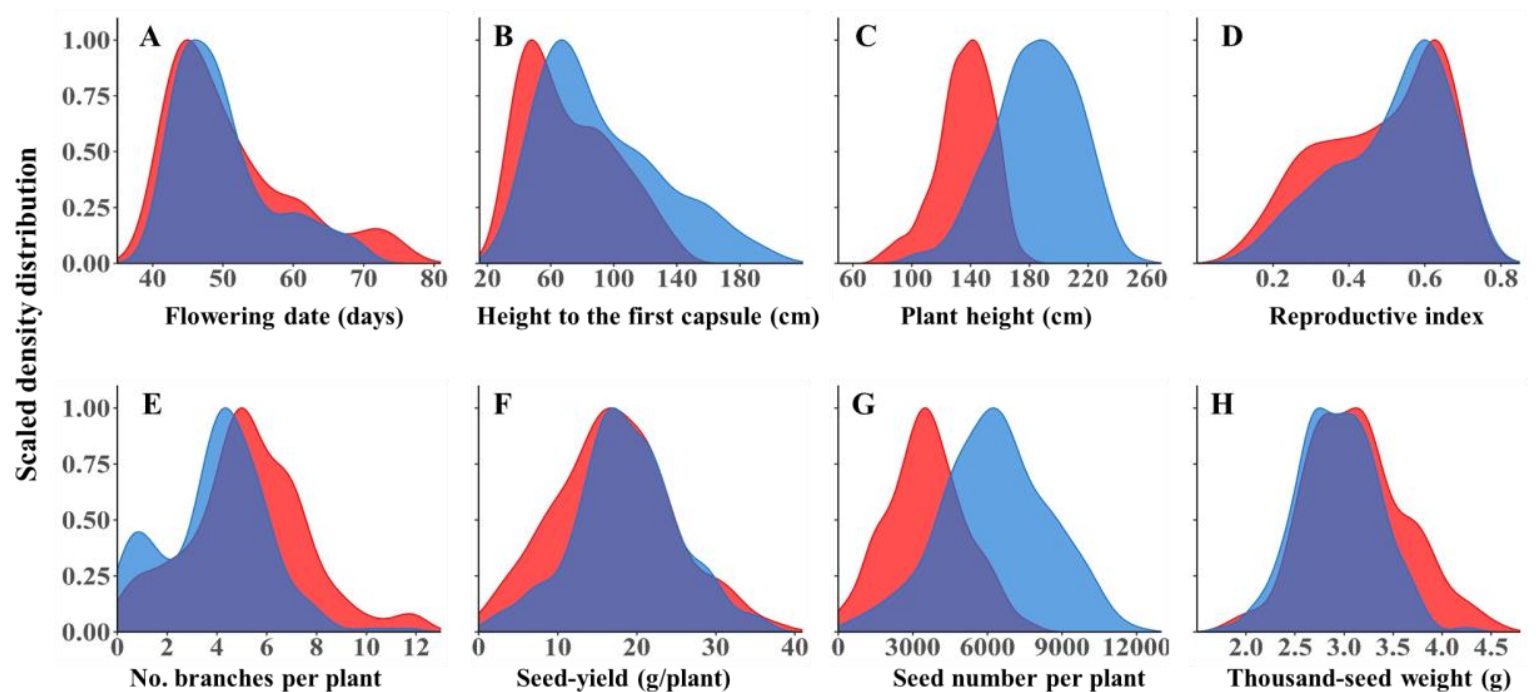

Figure 1. Density distribution of phenological traits: (A) Flowering date. Plant architecture traits: (B) height to the first capsule, (C) plant height, (D) reproductive index, and (E) number of branches per plant. Yield components: $(\mathbf{F})$ seed yield per plant, $(\mathbf{G})$ number of seeds per plant, and $(\mathbf{H})$ thousand-seed weight, under two years: 2018 (red) and 2020 (blue).
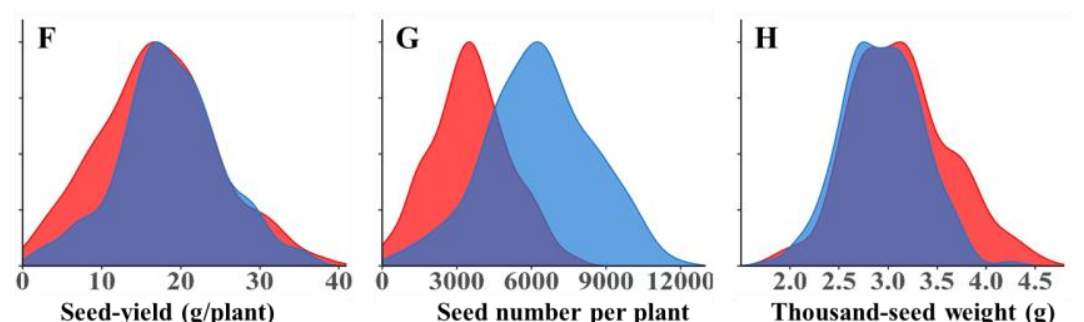

To test the effect of genotype $(\mathrm{G})$, year (Y), and genotype-by-year interaction $(\mathrm{G} \times \mathrm{Y})$, we estimated the variance component of each parameter for all the traits. In general, genotype and year had significant effects for most of the traits, with a significant $\mathrm{G} \times \mathrm{Y}$ interaction. The estimates 
Table 1. Variance component estimates for the random effects. $\sigma^{2}{ }_{G}$ is the genetic variance, $\sigma^{2} \mathrm{Y}$ is the year variance, $\sigma_{\mathrm{G} \times \mathrm{Y}}^{2}$ is the genotype-by-year variance, $\sigma_{\epsilon}^{2}$ is the error variance, and $H^{2}$ is the broad-sense heritability. Flowering date (FD), height to the first capsule (HTFC), plant height (PH), number of branches per plant (NBPP), reproductive index (RI), seed yield per plant (SYPP), number of seeds per plant (SNPP), and thousand-seed weight (TSW).

291

\begin{tabular}{|c|c|c|c|c|c|}
\hline \multirow{2}{*}{ Trait } & \multicolumn{4}{|c|}{ Variance component } & \multirow[b]{2}{*}{$H^{2}$} \\
\hline & $\sigma^{2} G$ & $\sigma^{2} Y$ & $\sigma^{2} G \times Y$ & $\sigma_{\epsilon}^{2}$ & \\
\hline FD & $61.48 * * *$ & $0.39^{\text {n.s. }}$ & $2.77 * * *$ & 8.84 & 0.97 \\
\hline HTFC & $1069 * * *$ & $214.6 * * *$ & $71.49 * * *$ & 125.95 & 0.96 \\
\hline PH & $441.81 * * *$ & $1174.8 * * *$ & $44.18 * * *$ & 289.12 & 0.89 \\
\hline $\mathbf{R Z}$ & $356.97 * * *$ & $394.51 * * *$ & $31.97 * * *$ & 204.56 & 0.91 \\
\hline $\mathbf{R I}$ & $0.02 * * *$ & $0.0003 *$ & $0.0009 * * *$ & 0.003 & 0.97 \\
\hline NBPP & $4.04 * * *$ & $0.72 * *$ & $0.64 * * *$ & 2.83 & 0.88 \\
\hline SYPP & $28.36 * * *$ & $0.72^{\text {n.s. }}$ & $10.21 * * *$ & 54.66 & 0.74 \\
\hline SNPP & $1723700.7 * * *$ & $3696689.4 *$ & $854375.3 * * *$ & 5260739 & 0.66 \\
\hline TSW & $0.17 * * *$ & $0.02 * * *$ & $0.03 * * *$ & 0.05 & 0.88 \\
\hline
\end{tabular}

n.s., $*$ and $* * *$ indicate non significant, significant differences at $P \leq 0.05$ and 0.001 , respectively, as determined by the likelihood ratio test.

To examine the relationship between vegetative-related traits (late FD, PH, and NBPP) and yield-related traits (RI and yield components), we applied PCA to BLUEs from the combined data. Based on this analysis, three PCs (eigenvalues > 1) accounted for $75.1 \%$ of the total phenotypic variance among the genotypes (Fig. 2A). PC1 explained 54.3\% of the variation, loaded positively to FD, HTFC, PH, and NBPP, and negatively loaded to RI, SNPP, SYPP, and TSW. PC2 explained $20.8 \%$ of the variation and loaded positively to HTFC, PH, NBPP, SYPP, SNPP, and TSW and negatively loaded to FD and RI. SYPP was positively correlated with SNPP (r=0.86) and TSW $(\mathrm{r}=0.37)$, however, no significant relationship was observed between these two traits ( $\mathrm{r}=-0.04$; Supplemental Table S4). Overall, FD and PH were positively correlated ( $\mathrm{r}=0.7)$ and both were negatively correlated with RI (r=-0.89 and r=-0.68, respectively; Fig. 2A; Supplemental Table S4). 

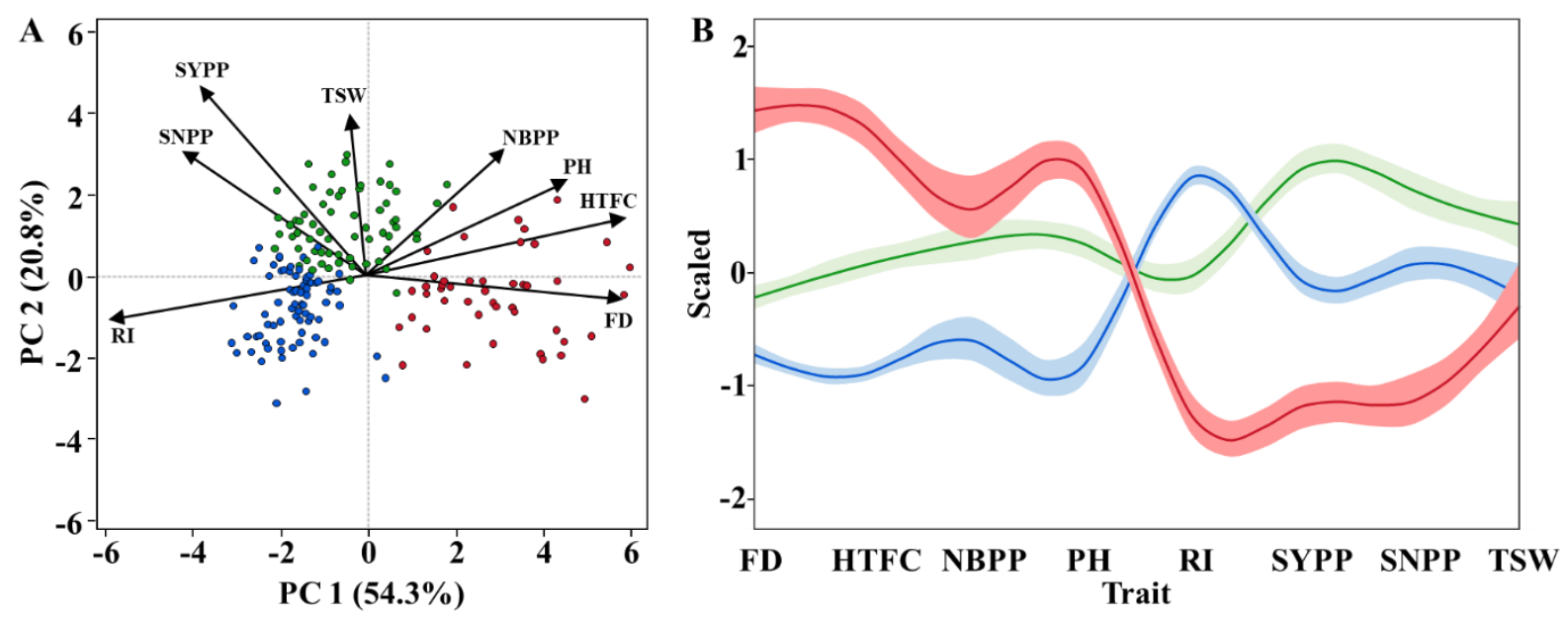

Figure 2. (A) Principal component (PC) analysis of phenotypic traits of the combined data (2018 and 2020). Each dot represents one genotype. (B) K-means clustering analysis of the primary traits. Y-axis is the centered and scaled values for each trait and the lines are the cluster means with their confidence intervals. Clusters 1 (Green), 2, (Blue), and 3 (red) were estimated using the K-means clustering analysis. The traits included Flowering date (FD), height to the first capsule (HTFC), plant height (PH), number of branches per plant (NBPP), reproductive index (RI), seed yield per plant (SYPP), number of seeds per plant (SNPP), and thousand-seed weight (TSW).

\subsection{Flowering date affects the final seed-yield via morphological modifications}

To further dissect the relationship between flowering date, plant architecture, and yield components, we applied k-means clustering analysis using the combined data. This analysis partitioned the panel into three clusters: early flowering (average 44.84 DAS), mid flowering (48.76 DAS), and late flowering (61.65 DAS) (Fig. 2B; Supplemental Table S5). Comparison between clusters 2 and 1 (early and mid-flowering) shows that they are different in terms of morphological and yield components. Although cluster 2 genotypes have larger RI ( 0.63 vs. 0.5), genotypes from cluster 1 had greater PH and NBPP (142.23 vs. $166.69 \mathrm{~cm}$ and 3.29 vs. 5.12 branches per plant, respectively). These alternations in the plant architecture traits affect the outcome, as we observed higher SYPP, SNPP, and TSW for cluster 1 genotypes (Fig. 2B; Supplemental Table S5). When we compared clusters 1 and 2 to cluster 3, we observed a major difference in FD (61.65 days) that led to a long vegetative phase and higher PH $(181.77 \mathrm{~cm})$ and NBPP (5.67), but these cluster 3 genotypes had lower RI (0.31) and lower SNPP, SYPP, and TSW performance. 


\subsection{Allelic diversity and population structure of the sesame panel}

333 To examine the genetic diversity at the genomic level, we used 20,294 SNP markers, spread along

334 the sesame genome. Principal component analysis on the SNP data explained $30.8 \%$ of the genetic variation between genotypes. The PCA did not show any separation between genotypes relative to their geographical origins (Fig. 3A). ADMIXTURE analysis partitioned the panel into four major (K1, K5, K6, and K7) and three minor (K2, K3, and K4) sub-populations (Fig. 3B). Fst values of these seven sub-populations ranged from 0.08 to 0.32 , which showed a weak to moderate differentiation among the subpopulations (Supplemental Table S6). Genome-wide LD analysis showed that LD decayed rapidly to half of its initial value (0.22) at 58,774 base pairs (Supplemental 341 Fig. S2).
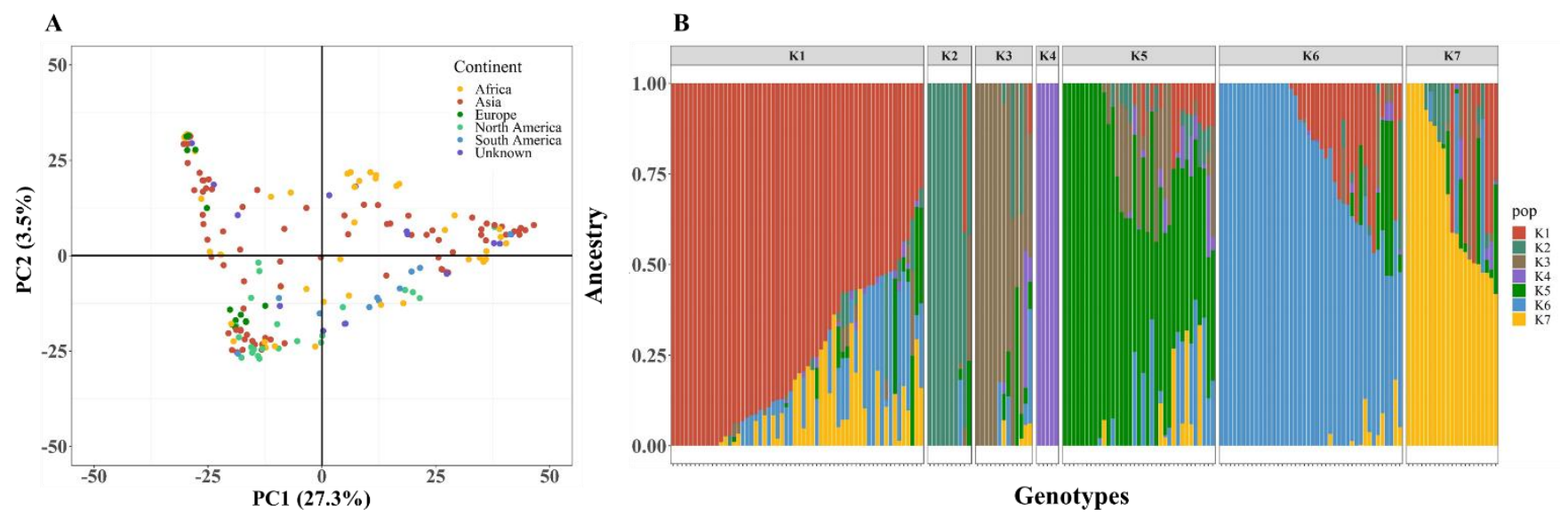

Figure 3. Population structure of the SCHUJI panel. (A) Principal component analysis and (B) ADMIXTURE when $\mathrm{K}=7$. Every single dot or line represents an individual genotype.

\subsection{Genomic heritability and genomic correlations}

To elucidate the trait similarity at the genomic level, we computed genomic heritability for each trait and genomic correlations between the measured traits. Estimates of genomic heritability ranged from $0.37(\mathrm{PH})$ to $0.58(\mathrm{SYPP})$ presenting moderate values for all the traits (Supplemental Table S7). Figure 4 presents phenotypic (upper triangular elements) and genomic (lower triangular

351 showed similar patterns (Fig. 4; supplemental Tables S4, S8). FD was positively correlated with 352 HTFC and PH (0.88 and 0.58, respectively), while negatively correlated with morphological yield353 related traits, such as RZ and RI. HTFC was found to be positively correlated with PH (0.71) while negativity correlated with RZ (-0.75), RI (-0.96), SYPP (-0.56), and SNPP (-0.6). SYPP was 

less related (0.17).

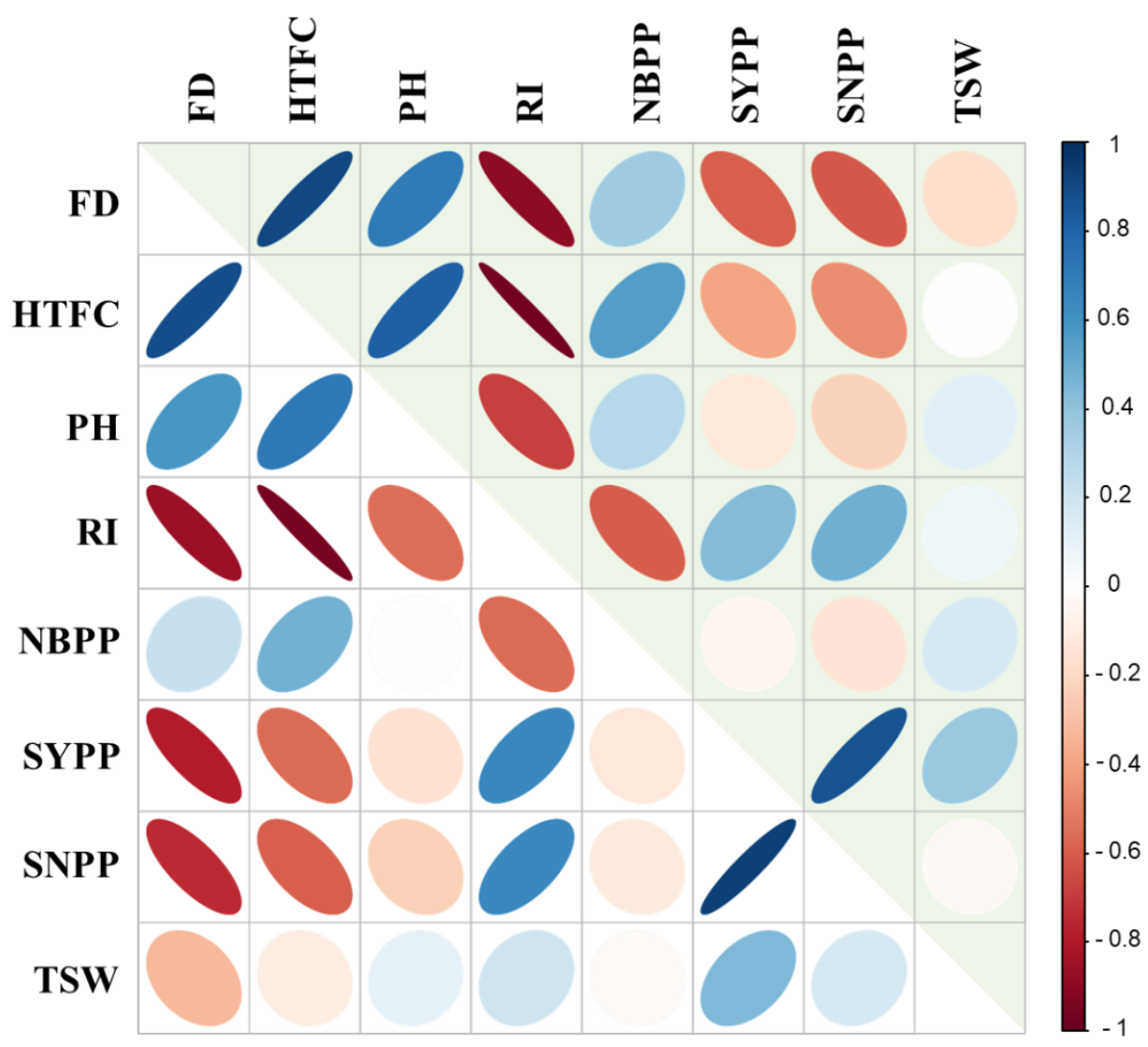

Figure 4. Heatmap of phenotypic (upper triangular elements) and genomic (lower triangular elements) correlation matrix between the primary traits: Flowering date (FD), height to the first capsule (HTFC), plant height $(\mathrm{PH})$, reproductive index (RI), number of branches per plant (NBPP),), seed yield per plant (SYPP), number of seeds per plant (SNPP), and thousand-seed weight (TSW). Colors indicate the level of correlations (r) from positive (blue) to negative (red).

\subsection{Association mapping of agronomic traits}

We performed a single-marker regression GWAS for all the traits and detected 11, 19, and 20 SNPs that are associated with trait mean differences for 2018, 2020, and combined data, respectively (Supplemental Table S9). For FD, we identified two major genomic regions on linkage group (LG) 2 and 11 (4, 4, and 5 SNPs for 2018, 2020 and combined data, respectively; Fig. 5A). In total, 9 SNPs were found significantly associated with the plant architecture traits, including 4 for HTFC no clear peak (Supplemental Fig. S3B-C). 


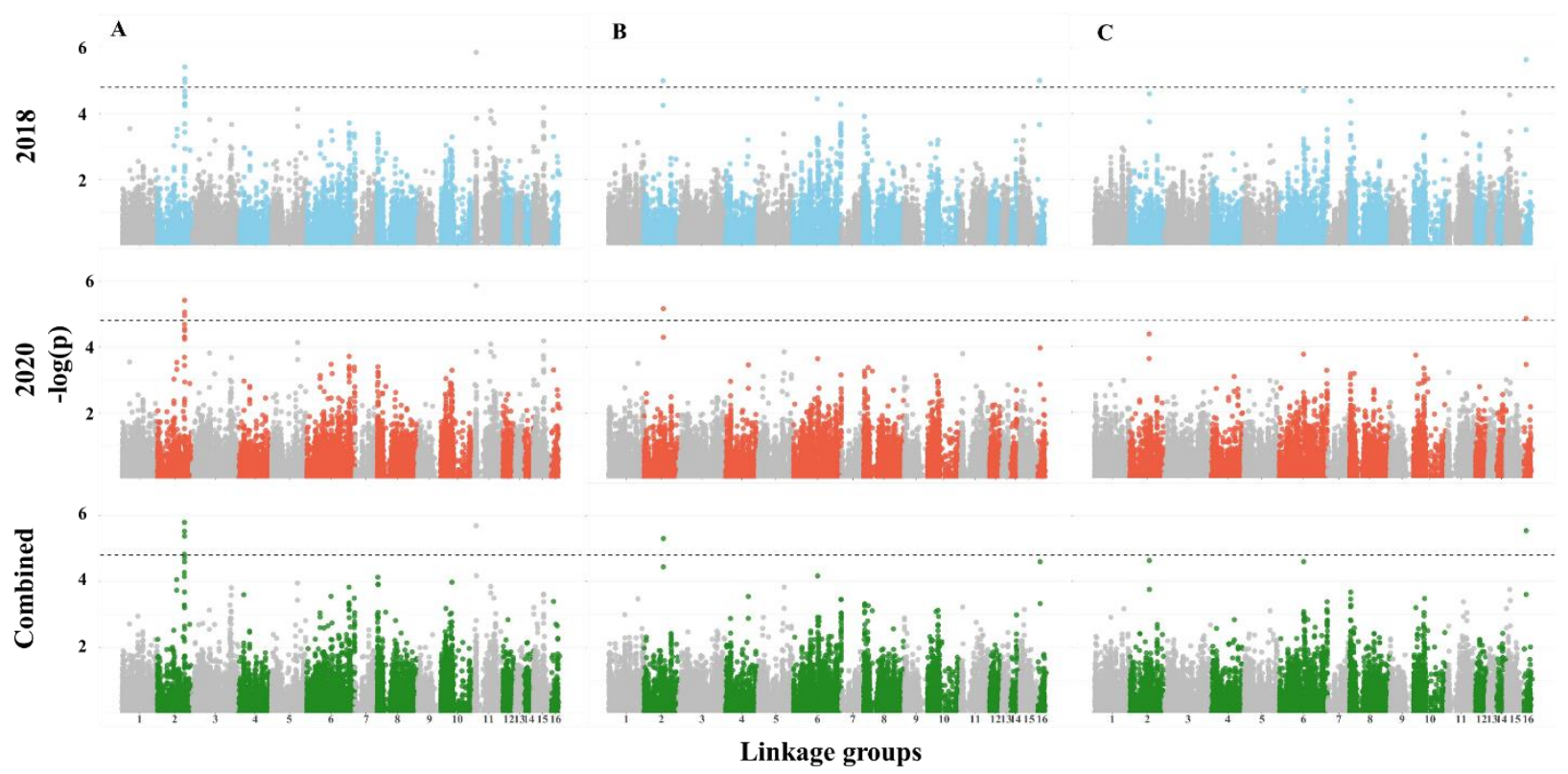

Figure 5. Manhattan plots for (A) flowering date, (B) height to the first capsule, and (C) reproductive index in 2018, 2020, and combined data. The dashed line represents the genome-wide significance threshold.

For SYPP and SNPP, we detected one major genomic region on LG2 (except for SNPP in 2018) (Fig. 6A-B). SYPP was associated with 20 SNPs while SNPP was associated with 7 SNPs (Supplemental Table S9). For TSW, we found one SNPs on LG1 that was slightly below the significant threshold $\left(-\log _{10}(p)=4.77\right)$, only in the 2020 season (Fig. 6C, and Supplemental Table S9). We compared the mapping results to find SNPs that overlap across traits. HTFC had one genomic region overlapping with RI on LG16 (Supplemental Table S9). FD had a major genomic region overlapping with SYPP and SNPP (Figs. 5-6; Supplemental Table S9). 


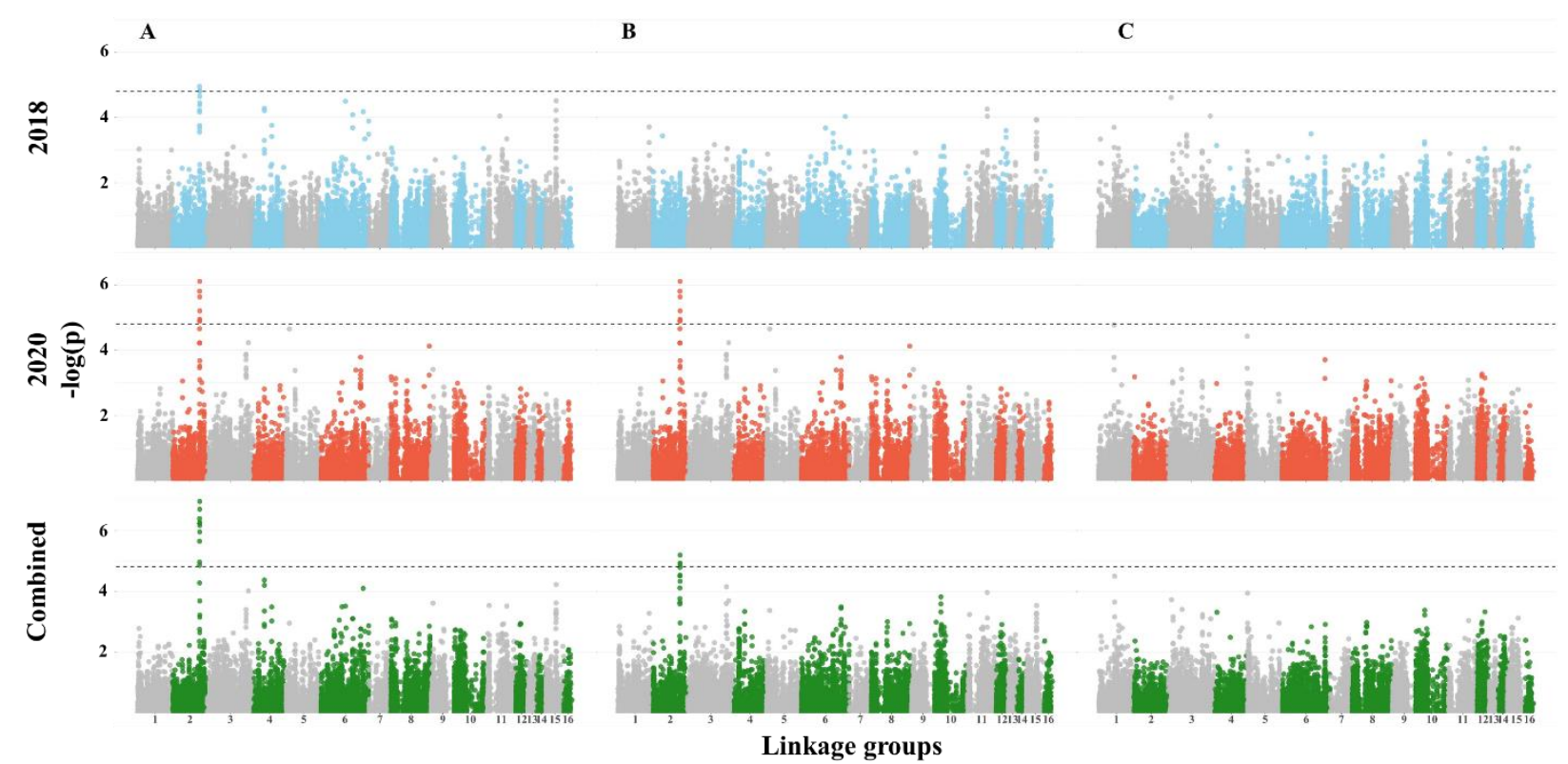

Figure 6. Manhattan plots for yield components traits: (A) seed yield per plant, (B) seed number per plant, and (C) thousand-seed weight. The dashed line represents the genome-wide significance threshold.

\subsection{Flowering date promotes yield stability in sesame}

A significant genomic region in the length of 96,491 base pairs that contained 10 SNPs on LG2 was found to be associated with FD, SNPP, and SYPP (Supplemental Table S9). In total, 4 SNPs inside this region overlapped with FD and SYPP and were within the range of LD-decay (Supplemental Fig. S2) in our panel. To explore their influence on the phenotypic variation of these two traits across the two growing seasons, we defined haplotypes for these 4 overlapping SNPs. Two possible haplotypes were found. The first haplotype (Hap1) was more frequent $(n=140)$ than the second haplotype (Hap2, n=30) (Fig. 7). Hap1 was found associated with earliness while genotypes that included Hap2 exhibited late flowering under the Mediterranean climate $(P<0.0001$, Fig. 7A). Moreover, these two haplotypes also differed in yield performance, with Hap1 promoting higher seed yield (Fig. 7B). To test the phenotypic stability of the haplotypes

423 FD and SYPP from BLUE per year analysis. While the haplotypes had a significant effect on the 424 traits $(P<0.0001$ for FD and SYPP), we did not observe any significant interaction between 425 haplotypes and year $(P=0.087$ for FD and $P=0.82$ for SYPP). These results may indicate that this genomic region promotes yield stability via modifications in FD. Moreover, we obtained 
A

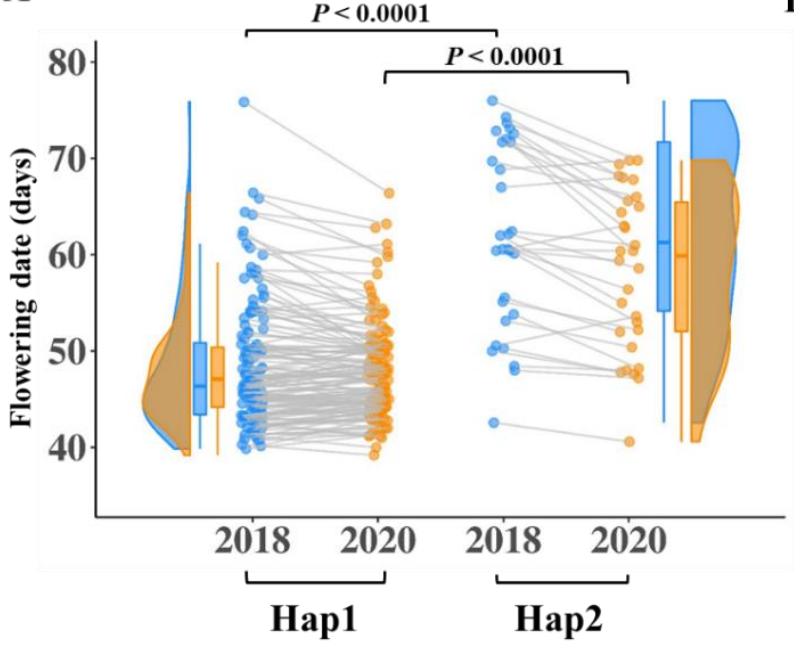
same genotypes between the two growing seasons (Fig. 7B).

years. For FD, we found that genotypes in both haplotypes had a similar pattern $(P=0.5$ and $P=0.12$, for year effect) as expressed in parallel trend lines between genotypes across years (Fig. 7A). For SYPP, the differences in mean values across years were on the edge of statistical significance $(P=0.055$ and $P=0.32$ for Hap1 and Hap2, respectively), but the genotypes within each haplotype had different values across years as expressed with the crossing lines that connected the al., 2019).
B

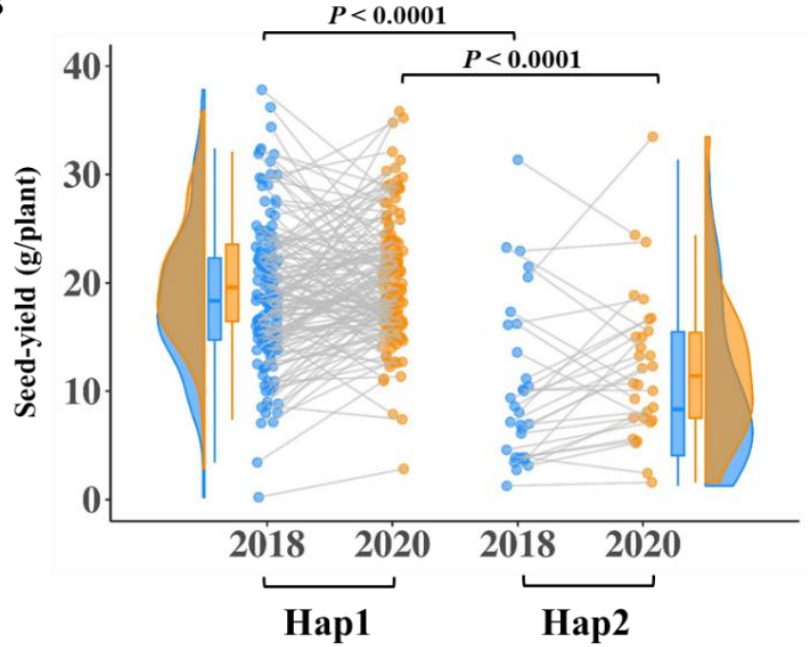

Figure 7. Haplotype analysis of the major genomic region on LG2 and their effects on (A) flowering date and (B) seed yield across two years. Every dot represents a genotype. Blue color represents the 2018 growing season, orange represents the 2020 growing season, and grey lines connect the same genotypes in the two growing seasons.

To find the genetic basis underlying the biological influence on the traits, we searched for candidate genes (CGs) within this LG2 hotspot genomic region. Overall, we found 20 CGs: 7 CGs were located inside of the genomic region, while 13 CGs were consisted of upstream (8) and downstream (5) of the genomic region. (Supplemental Table S10). Although four of the CGs encode uncharacterized proteins, most of the CGs were involved in signaling pathways and regulation and four of them (LOC105156148, LOC105156152, LOC105156159, and LOC105156313) were associated with controlling flowering time, floral development, and productivity in Arabidopsis and rice (Ito et al., 2007; Wu et al., 2012; Miao et al., 2018; Teng et 


\section{DISCUSSION}

452 Understanding the genetic basis of agronomic traits is important for research and breeding efforts.

453 Here, we explored the genetic and phenotypic variation in a newly established sesame panel 454 (SCHUJI) aiming at dissecting the genomic architecture of morpho-agronomic traits. We 455 hypothesized that as 'orphan crop-plant' sesame has not been subjected to modern selection, the 456 geographically distributed panel preserved an ample allelic repertoire for agronomically important 457 traits. Evaluation of the level and extent of genetic diversity among the sesame panel surprisingly 458 showed that there is no clear geographical separation between genotypes (Fig. 3A). Similarly, 459 previous genetic characterization of other sesame panels showed no clustering associated with 460 continents and suggested sub-geographical regions or latitudes as key factors (Wei et al., 2015; Cai 461 et al., 2016; Dossa et al., 2016). Moreover, the ADMIXTURE analysis resulted in seven sub462 populations $(\mathrm{K}=7)$ with a low to moderate diffrrences between them (Fig. 3B; Supplemental Table 463 S6). Thus, the lack of geographic signature in our, as well as other genetic panels, may be a 464 consequence of recent genetic materials exchange (Basak et al., 2019) or unavailable information 465 at the collection sites. As an example, even the smallest sub-populations $(2,3$, and 4) that were 466 more genetically uniform showed the various origin of genotypes (i.e., sub-population 4 included 467 genotypes from Thailand, Israel, or unknown) (Fig. 3B).

471 High phenotypic diversity was found for all morpho-agronomical traits across the two years, with 4722020 expressing higher values (Fig. 1). This observation agrees with previous studies using other 473 sesame panels (Furat \& Uzun, 2010; Zhou et al., 2018). In general, FD was found to be negatively 474 correlated with yield components, such as RI, SYPP, and SNPP. On the other hand, late FD was 475 associated with high NBPP, HTFC, and PH (Figs. 2, 4; Supplemental Table S4). Additional 476 support for the key effect of FD on productivity comes from the k-means clustering analysis. This 477 analysis clustered the panel into three groups (Fig. 2B), which were also associated with the genetic 478 structure of the panel (Supplemental Fig. S4). Comparison between the mid-FD group (green) and the early FD (blue) showed that NBPP and PH have a positive effect on productivity traits, which 
that increasing the source size (i.e., vegetative organs) can support a higher seed set and filling. Thus, the higher yield potential of cluster 1 can be a consequence of greater vegetative biomass accumulation following more branches. Interestingly, the late-flowering group (red) had a similar number of branches as the intermediate group (green); however, most of them were not fertile due to the late-flowering phenotype. RI is a key trait for yield potential assessment and represents the ratio between RZ and PH. As expected, RI and FD are negatively correlated (Fig. 2). However, it is worth noted that a high value of RI can be a consequence of either high PH with low HTFC or small plants. Therefore, to promote higher yield potential, it is important to combine high RI with high RZ values.

Langham (2007) reported that photoperiod responsiveness plays a key role in sesame flowering date and vegetative biomass accumulation. Our results show that while most genotypes that belong to the late FD and low RI group clustered together, they differ in their geographical origins (Fig. 3A; Supplemental Fig. S4), which may suggest the involvement of other genetic and/or enviomental factors.

\subsection{Genetic architecture of agronomical traits reveals hotspot of overlapping genomic regions}

To detect the genetic basis underlying observed phenotypic variation, we conducted single-marker regression GWAS. Overall, we detected 50 associated SNPs for all the traits, with 11 for 2018 and 19 for 2020 seasons, and 20 for the combined data that were spread along the entire sesame genome. The flowering date was mapped to two genomic regions (13 SNPs) on LGs 2 and 11 (Fig. 5A and Supplemental Table S9). The major genomic region on LG2 detected in the current study under the Mediterranean climate was previously reported in another sesame panel, however, it was less significant under other environments (Wei et al., 2015). The advantage of using a diversity panel to detect new alleles is exemplified by comparing our results with a bi-parental population that was grown under the same environmental conditions. While Teboul et al. (2020) detected six QTLs using the $\mathrm{F}_{2}$ population $(\mathrm{S}-91 \times \mathrm{S}-297)$, only the genomic region on LG11 was overlap with the current study.

The plant architecture traits were associated with 9 SNPs [HTFC (4), RZ (2), and RI (3)], with some overlaps (Fig. 5B, 5C; Supplemental Fig. S4A; Supplemental Table S9). Two genomic regions on LG 2 and 16 showed overlaps for HTFC and RI, which correspond to a high genomic correlation $(r=-0.96)$ between those traits. These results suggest that genotypes with shorter HTFC 
will have the potential to extend the growth period and develop more capsules (i.e., high RI). Notably, while FD and PH showed positive phenotypic and genomic correlations $(0.70$ and 0.58 , respectively), we did not detect any genomic region associated with $\mathrm{PH}$. On the other hand, clustering analysis of the phenotypic data suggested that different genotypes with similar PH can differ in FD. Thus, the lack of genomic region associated with PH may result in either many small effect loci or management effect. It is also worth noting that since sesame is grown as a summer crop under irrigation management and characterized with indeterminate growth habits, it might affect the diversity of this trait (Supplemental Table S3).

Twenty-eight SNPs were significantly associated with yield components. Of those, 20 were associated with SYPP, 7 for SNPP, and 1 for TSW. Seed size (TSW) is known to have moderatehigh heritability in sesame (Uzun et al., 2013; Kalaiyarasi et al., 2019), as was found in the current study (0.88) and other crops, such as wheat (Sukumaran et al., 2018) and pea (Pisum sativum L.; Huang et al., 2017), was associated with only one region in 2020, with no overlap with SNPP and SYPP. Likewise, a small number of associated loci for TSW were found in bi-parental sesame populations (Du et al., 2019; Teboul et al., 2020), which may suggest that this trait is under less complex genetic control or under the regulation of many small-effect genomic regions. The absence of significant genomic signs for TSW (as well as other traits in the current study) indicates that other approaches, such as genomic prediction (Crossa et al., 2017), may contribute to understanding its genetic basis. The positive phenotypic and genomic correlations between TSW and SYPP (0.37 and 0.44, respectively), and between SNPP and SYPP (0.86 and 0.93) on one side, and the absence of both correlations between TSW and SNPP (-0.04 and 0.17, respectively) on the other side, open up the possibility to breed simultaneously for both traits and improve yield.

SYPP and SNPP are highly polygenic traits associated with various anatomical and morphological traits (e.g., NBPP, RZ, RI, PH, number of capsules per plant, number of capsules per leaf axil, carpel number per capsule, and seed size). For SNPP and SYPP, we found one shared genomic region on LG2 overlapping with FD (Supplemental Table S9). This demonstrates the important role of FD on productivity, which is supported by the negative phenotypic and genomic 539 correlations between these traits (Fig. 4). The effect of flowering on seed yield can be also attributed to the in-determine growth habit of sesame, and the agronomic practices to stop the irrigation to harvest the crop before it rains in autumn. Under such conditions, plants that were 542 able to flower earlier had a longer period to produce flowers and capsules and obtain a higher yield. 
543 In contrast, genotypes that flowered later in the season were more exposed to environmental

544 conditions. A similar pattern was found in other in-determined crops such as soybean (Zhang et

545 al., 2015) and chickpea (Cicer arietinum L.; Upadhyaya et al., 2015). It is worth noted that while

546 FD had high broad-sense heritability (0.97), both SYPP and SNPP had relatively lower broad-sense

547 heritability estimates (0.74 and 0.66, respectively) (Table 1$)$. These results, along with haplotypes

548 analysis (Fig. 7), suggest that these yield components are controlled by other less heritable factors

549 not related to FD.

550 In sesame, allelic variation within flowering-related genes contributes to variation in 551 flowering date (Wei et al., 2016; Zhou et al., 2018). In the current study, we found two genomic 552 regions (LGs 2 and 11) that were associated with FD across years. The significant marker on LG11 553 is located within LOC105173174, which encodes to AT-hook motif nuclear-localized protein 9. 554 The members of this gene family are associated with the regulation of flowering date in other plants 555 species (Zhao et al., 2014). The co-localization of the major genomic region on LG2 for FD and 556 yield components together may suggest that this region contains one major gene with a pleiotropic 557 effect or cluster of several genes. Analysis of CGs within this genomic region highlighted 20 558 flowering and productivity-related genes (Supplemental Table S10). LOC105156148 is encoding 559 nitrate transporter (NRT1) and was found in Arabidopsis to interact with two flowering regulators 560 transcription factors, CONSTANS and FLOWERING LOCUS C (FLC) (Teng et al., 2019). 561 LOC105156159 is encoding abscisic acid receptor PYRl-like, a mutant allele of this gene is found 562 to be associated with growth and productivity in rice (Miao et al., 2018). Two of the significant 563 markers within this genomic region were within the LOC105156152 gene that encodes 564 CHROMATIN REMODELING 19 regulating floral organs in Arabidopsis (Wu et al., 2012). The 565 functional annotation of these genes and the co-localization of them in the same genomic region 566 demonstrated how the variation in FD and SYPP could be genetically controlled together. Further 567 investigation is needed to study how this genomic region (and the genes inside it) interact with 568 other identified genomic regions for a deeper understanding of the genetic basis and mechanisms 569 underlying FD and SYPP variations in sesame. 


\section{Conclusion for future perspective}

572 While sesame is still mostly grown under a traditional cropping system, future sesame breeding 573 targets should focus on improving yield and adaptively to more diverse environments. Thus, 574 elucidating the genetic architecture controlling phenological, morphological, and yield components 575 traits will aid in understanding selection criteria and better genetic-based breeding. Here we 576 established a new sesame panel (SCHUJI) and explored its genetic variation for morpho-agronomic 577 traits under the Mediterranean climate conditions. We showed the benefits of using the globally 578 distributed panel for discovering new alleles associated with these traits. A major genomic region on LG2 was found in association with the flowering date and yield components, indicating the crucial role of phenology on sesame production. A better understanding of the genetic variability underlying flowering date in sesame will serve as a basis for improving sesame adaptively to new crop from domestically grown to global production in intensive agriculture.

\section{ACKNOWLEDGEMENTS}

We highly appreciate the excellent technical support of the Peleg lab members. This research was partly supported by the Chief Scientist of the Israel Ministry of Agriculture and Rural Development (837-0150-14), and The Hebrew University of Jerusalem.

\section{REFERENCES}

Alexander, D. H., Novembre, J., \& Lange, K. (2009). Fast model-based estimation of ancestry in unrelated individuals. Genome Research, 19(9), 1655-1664.

Allen, M., Poggiali, D., Whitaker, K., Marshall, T. R., van Langen, J., \& Kievit, R. A. (2021). Raincloud plots: a multi-platform tool for robust data visualization.

Anilakumar, K. R., Pal, A., Khanum, F., \& Bawa, A. S. (2010). Nutritional, medicinal and industrial uses of sesame (Sesamum indicum L.) seeds - An overview. Agriculturae Conspectus Scientificus, 75(4), 159-168. 
601

mapping and genome-wide association analysis identifies candidate genes for phytophthora blight resistance in sesame (Sesamum indicum L.). Frontiers in Plant Science, 12, 604709.

Basak, M., Uzun, B., \& Yol, E. (2019). Genetic diversity and population structure of the Mediterranean sesame core collection with use of genome-wide SNPs developed by double digest RAD-Seq. PLOS ONE, 14(10), e0223757.

Bates, D., Mächler, M., Bolker, B., \& Walker, S. (2015). Fitting linear mixed-effects models using lme4. Journal of Statistical Software 67(1), 1-48.

Baydar, H. (2005). Breeding for the improvement of the ideal plant type of sesame. Plant Breeding, 124(3), 263-267.

Bedigian, D. (2015). Systematics and evolution in Sesamum L. (Pedaliaceae), part 1: Evidence regarding the origin of sesame and its closest relatives. Webbia, 70(1), 1-42.

Bradbury, P. J., Zhang, Z., Kroon, D. E., Casstevens, T. M., Ramdoss, Y., \& Buckler, E. S. (2007). TASSEL: software for association mapping of complex traits in diverse samples. Bioinformatics, 23(19), 2633-2635.

Browning, S. R., \& Browning, B. L. (2007). Rapid and accurate haplotype phasing and missingdata inference for whole-genome association studies by use of localized haplotype clustering. American Journal of Human Genetics, 81(5), 1084-1097.

Cai, G., Yang, Q., Chen, H., Yang, Q., Zhang, C., Fan, C., \& Zhou, Y. (2016). Genetic dissection of plant architecture and yield-related traits in Brassica napus. Scientific Reports, 6(1), 1-16.

Crossa, J., Pérez-Rodríguez, P., Cuevas, J., Montesinos-López, O., Jarquín, D., de los Campos, G., Burgueño, J., González-Camacho, J. M., Pérez-Elizalde, S., Beyene, Y., Dreisigacker, S., Singh, R., Zhang, X., Gowda, M., Roorkiwal, M., Rutkoski, J., \& Varshney, R. K. (2017). Genomic selection in plant breeding: methods, models, and perspectives. Trends in Plant Science 22(11), 961-975.

Danecek, P., Auton, A., Abecasis, G., Albers, C. A., Banks, E., DePristo, M. A., Handsaker, R. E., Lunter, G., Marth, G. T., Sherry, S. T., McVean, G., \& Durbin, R. (2011). The variant call format and VCFtools. Bioinformatics, 27(15), 2156-2158.

Delgado, M. \& Yermanos, D. M. (1975). Yield components of sesame (Sesamum Indicum L.) under different population densities. Economic Botany, 29(1), 69-78.

De los Campos, G., Sorensen, D., \& Gianola, D. (2015). Genomic heritability: What is it? PLoS Genetics, 11(5), 1-21. 
Dossa, K., Diouf, D., Wang, L., Wei, X., Zhang, Y., Niang, M., ... Cisse, N. (2017). The emerging oilseed crop Sesamum indicum enters the "Omics" era. Frontiers in Plant Science, $8,1154$.

Dossa, K., Li, D., Zhou, R., Yu, J., Wang, L., Zhang, Y., ... Zhang, X. (2019). The genetic basis of drought tolerance in the high oil crop Sesamum indicum. Plant Biotechnology Journal, 17(9), 1788-1803.

Dossa, K., Wei, X., Zhang, Y., Fonceka, D., Yang, W., Diouf, D., ... Zhang, X. (2016). Analysis of genetic diversity and population structure of sesame accessions from Africa and Asia as major centers of its cultivation. Genes, 7(4), 14.

Doyle, J. \& Doyle, J. 1987. Genomic plant DNA preparation from fresh tissue-CTAB method. Phytochem Bull, 19, 11-15.

Du, H., Zhang, H., Wei, L., Li, C., Duan, Y., \& Wang, H. (2019). A high-density genetic map constructed using specific length amplified fragment (SLAF) sequencing and QTL mapping of seed-related traits in sesame (Sesamum indicum L.). BMC Plant Biology, 19(1), 1-20.

Elshire, R. J., Glaubitz, J. C., Sun, Q., Poland, J. A., Kawamoto, K., Buckler, E. S., \& Mitchell, S. E. (2011). A Robust, simple genotyping-by-sequencing (GBS) approach for high diversity species. PLOS ONE, 6(5), e19379.

Endelman, J. B. (2011). Ridge regression and other kernels for genomic selection with R package rrBLUP. The Plant Genome, 4(3), 250-255.

Furat, S., \& Uzun, B. (2010). The use of agro-morphological characters for the assessment of genetic diversity in sesame (Sesamum indicum L.). Plant Omics Journal, 3(3), 85-91.

Gadri, Y., Eshed Williams, L., \& Peleg, Z. (2020). Tradeoffs between yield components promote crop stability in sesame. Plant Science, 295, 110105.

Guo, Z., Chen, D., Alqudah, A. M., Röder, M. S., Ganal, M. W., \& Schnurbusch, T. (2017). Genome-wide association analyses of 54 traits identified multiple loci for the determination of floret fertility in wheat. New Phytologist, 214(1), 257-270.

Henderson, C. R. (1975). Best linear unbiased estimation and prediction under a selection model. Biometrics, 31(2), 423.

Hill, W. G., \& Weir, B. S. (1988). Variances and covariances of squared linkage disequilibria in finite populations. Theoretical Population Biology, 33(1), 54-78.

Huang, S., Gali, K. K., Tar'an, B., Warkentin, T. D., \& Bueckert, R. A. (2017). Pea phenology: 
crop potential in a warming environment. Crop Science, 57(3), 1540-1551.

Ito, T., Nagata, N., Yoshiba, Y., Ohme-Takagi, M., Ma, H., \& Shinozaki, K. (2007). Arabidopsis MALE STERILITY1 encodes a PHD-Type transcription factor and regulates pollen and tapetum development. The Plant Cell, 19, 3549-3562.

Kalaiyarasi, R., Lokeshkumar, K., Mohanraj, M., Priyadharshini, A. \& Rajasekar, R. (2019). Genetic variability parameters for yield and yield related traits in sesame (Sesamum indicum L.). International Journal of Current Microbiology and Applied Sciences, 8(8), 819-825.

Kassambara, A. \& Mundt, F. (2020). factoextra: Extract and Visualize the Results of Multivariate Data Analyses. https://CRAN.R-project.org/package=factoextra linear mixed effects models . Journal of Statistical Software, 82(13), 1-26.

Langham, R. (2007). Phenology of Sesame. New Crops and New Uses, 144-182. (2018). GWAS uncovers differential genetic basis for drought and salt tolerances in sesame at the germination stage. Genes, $9(2), 87$.

Li, H., Peng, Z., Yang, X., Wang, W., Fu, J., Wang, J., Han, Y., Chai, Y., Guo, T., Yang, N., Liu, J., Warburton, M. L., Cheng, Y., Hao, X., Zhang, P., Zhao, J., Liu, Y., Wang, G., Li, J., \& Yan, J. (2012). Genome-wide association study dissects the genetic architecture of oil

Mei, H., Liu, Y., Du, Z., Wu, K., Cui, C., Jiang, X., Zhang, H., \& Zheng, Y. (2017). High-density biosynthesis in maize kernels. Nature Genetics, 45(1), 43-50.

Li, J., \& Ji, L. (2005). Adjusting multiple testing in multilocus analyses using the eigenvalues of a correlation matrix. Heredity, 95(3), 221-227.

Marroni, F., Pinosio, S., Zaina, G., Fogolari, F., Felice, N., Cattonaro, F., \& Morgante, M. genetic map construction and gene mapping of basal branching habit and flowers per leaf axil in sesame. Frontiers in Plant Science, 8, 636.

Miao, C., Xiao, L., Hua, K., Zou, C., Zhao, Y., Bressan, R. A., \& Zhu, J.K. (2018). Mutations in a subfamily of abscisic acid receptor genes promote rice growth and productivity. Proceedings of the National Academy of Sciences of the United States of America, 15(23), 6058-6063. 
694

695

696

697

698

699

700

701

702

703

704

705

706

707

708

709

710

711

712

713

714

715

716

717

718

719

720

721

722

723

724

Miao, H., Li, C., Duan, Y., Wei, L., Ju, M., \& Zhang, H. (2020). Identification of a Sidwf1 gene controlling short internode length trait in the sesame dwarf mutant dw607. Theoretical and Applied Genetics, 133(1), 73-86.

Mushtaq, A., Hanif, M. A., Ayub, M. A., Bhatti, I. A., \& Jilani, M. I. (2020). Sesame. In Medicinal Plants of South Asia (pp. 601-615). Elsevier.

Pérez, P., \& De Los Campos, G. (2014). Genome-wide regression and prediction with the BGLR statistical package. Genetics, 198(2), 483-495.

Purcell, S., Neale, B., Todd-Brown, K., Thomas, L., Ferreira, M. A. R., Bender, D., Maller, J., Sklar, P., De Bakker, P. I. W., Daly, M. J., \& Sham, P. C. (2007). PLINK: A tool set for whole-genome association and population-based linkage analyses. American Journal of Human Genetics, 81(3), 559-575.

R Core Team (2020). R: A language and environment for statistical computing. R Foundation for Statistical Computing, Vienna, Austria. https://www.R-project.org/.

Schmidt, P., Hartung, J., Bennewitz, J., \& Hans-Peter, P. (2019). Heritability in plant breeding on a genotype-difference basis. Genetics, 212(4), 991-1008.

Sonah, H., O’Donoughue, L., Cober, E., Rajcan, I., \& Belzile, F. (2015). Identification of loci governing eight agronomic traits using a GBS-GWAS approach and validation by QTL mapping in soya bean. Plant Biotechnology Journal, 13(2), 211-221.

Sukumaran, S., Lopes, M., Dreisigacker, S., \& Reynolds, M. (2018). Genetic analysis of multienvironmental spring wheat trials identifies genomic regions for locus-specific trade-offs for grain weight and grain number. Theoretical and Applied Genetics, 131(4), 985-998.

Teboul, N., Gadri, Y., Berkovich, Z., Reifen, R., \& Peleg, Z. (2020). Genetic architecture underpinning yield components and seed mineral-nutrients in sesame. Genes, 11(10), 1221.

Teng, Y., Liang, Y., Wang, M., Mai, H., \& Ke, L. (2019). Nitrate Transporter 1.1 is involved in regulating flowering time via transcriptional regulation of FLOWERING LOCUS C in Arabidopsis thaliana. Plant Science. 284, 30-36

Tibbs Cortes, L., Zhang, Z., \& Yu, J. (2021). Status and prospects of genome-wide association studies in plants. Plant Genome 14(1), e20077

Upadhyaya, H. D., Bajaj, D., Das, S., Saxena, M. S., Badoni, S., Kumar, V., Tripathi, S., Gowda, C. L. L., Sharma, S., Tyagi, A. K., \& Parida, S. K. (2015). A genome-scale integrated approach aids in genetic dissection of complex flowering time trait in chickpea. Plant 
Uzun, B., Yol, E., Furat, S. (2013). Genetic advance, heritability and inheritance in determinate growth habit of sesame. Australian Journal of Crop Science, 7(7), 978-983.

VanRaden, P. M. (2008). Efficient methods to compute genomic predictions. Journal of Dairy Science, 91(11), 4414-4423.

Wei, T \& Simko, V. (2017). R package "corrplot": Visualization of a correlation matrix (Version 0.84). https://github.com/taiyun/corrplot

Wei, X., Liu, K., Zhang, Y., Feng, Q., Wang, L., Zhao, Y., ... Zhang, X. (2015). Genetic discovery for oil production and quality in sesame. Nature Communications, 6(5), 8609.

Wei, X., Zhu, X., Yu, J., Wang, L., Zhang, Y., Li, D., Zhou, R., \& Zhang, X. (2016). Identification of sesame genomic variations from genome comparison of landrace and variety. Frontiers in Plant Science, 7, 1169.

Weir, B. S. \& Cockerham, C. C. (1984). Estimate F-statistics for the analysis of population structure. Evolution, 38(6), 1358-1370.

Wickham, H (2016). ggplot2: Elegant Graphics for Data Analysis. Springer-Verlag New York.

Wu, M.-F., Sang, Y., Bezhani, S., Yamaguchi, N., Han, S.-K., Li, Z., ... Jacobsen, S. E. (2011). SWI2/SNF2 chromatin remodeling ATPases overcome polycomb repression and control floral organ identity with the $L E A F Y$ and SEPALLATA3 transcription factors. Proceedings of the National Academy of Sciences of the United States of America, 109(9), 3576-3581.

Yu, J., Pressoir, G., Briggs, W. H., Vroh Bi, I., Yamasaki, M., Doebley, J. F., McMullen, M. D., Gaut, B. S., Nielsen, D. M., Holland, J. B., Kresovich, S., \& Buckler, E. S. (2006). A unified mixed-model method for association mapping that accounts for multiple levels of relatedness. Nature Genetics, 38(2), 203-208.

Zhang, J., Song, Q., Cregan, P. B., Nelson, R. L., Wang, X., Wu, J., \& Jiang, G. L. (2015). Genome-wide association study for flowering time, maturity dates and plant height in early maturing soybean (Glycine max) germplasm. BMC Genomics, 16(1), 1-11.

Zhao, J., Favero, D. S., Qiu, J., Roalson, E. H., \& Neff, M. M. (2014). Insights into the evolution and diversification of the AT-hook Motif Nuclear Localized gene family in land plants. BMC Plant Biology, 14(1), 266. Islam, M. R., Reynolds, A., Mezey, J., McClung, A. M., Bustamante, C. D., \& McCouch, S. 
R. (2011). Genome-wide association mapping reveals a rich genetic architecture of complex traits in Oryza sativa. Nature Communications, 2(1), 467.

758 Zhou, R., Dossa, K., Li, D., Yu, J., You, J., Wei, X., Zhang, X., Zhou, R., Dossa, K., Li, D., Yu,

759 J., You, J., Wei, X., \& Zhang, X. (2018). Genome-wide association studies of 39 seed yieldrelated traits in sesame (Sesamum indicum L.). International Journal of Molecular Sciences, 19(9), 2794.

762 Zhou, R., Liu, P., Li, D., Zhang, X., \& Wei, X. (2018). Photoperiod response-related gene SiCOL1 contributes to flowering in sesame. BMC Plant Biology, 18, 343. 